\title{
Cyclic steady state of simulated moving bed processes for enantiomers separation
}

\author{
Mirjana Minceva, Luis S. Pais, Alirio E. Rodrigues* \\ Laboratory of Separation and Reaction Engineering (LSRE), Faculty of Engineering, University of Porto, Rua Dr. Roberto Frias s/n, $4200-465$ Porto, \\ Portugal
}

Received 17 August 2001; received in revised form 25 February 2002; accepted 15 March 2002

\begin{abstract}
Simulated moving bed (SMB) technology developed by UOP in early 1960s has expanded greatly in the last decade, finding new applications in the area of natural products, fine chemistry and pharmaceutical industry. SMB processes are periodic processes designed to operate in cyclic steady state (CSS) and, therefore, the correct determination of CSS is needed for the assessment of the SMB performance. Two approaches can be used for determination of CSS: the dynamic simulation until CSS is reached and direct prediction of CSS. The direct prediction of CSS could be obtained in two ways: (i) considering that at CSS the spatially distributed SMB unit state at the end of the cycle is identical to that at its beginning (Method 1); or (ii) considering that at CSS the spatially distributed SMB unit state at the end of a switching time interval is identical to the state at the beginning of the interval, apart from a shift of exactly one column length (Method 2). The mathematical models assume axial dispersion flow and linear driving force (LDF) approximation for intraparticle mass transfer. Mathematical models were solved using the gPROMS (general Process Modelling System) software package. Both approaches (dynamic simulation and direct CSS prediction) were applied to the prediction of cyclic steady state of SMB unit for 1,1'-bi-2-naphthol enantiomers separation. The direct CSS predictions were compared with the standard dynamic simulation CSS prediction in terms of accuracy of SMB performance and computing time requirements; the Method 2 for CSS prediction is more efficient than the standard dynamic simulation.
\end{abstract}

(C) 2002 Elsevier Science B.V. All rights reserved.

Keywords: Simulated moving bed; Direct cyclic steady state prediction; Dynamic simulation; Enantiomers separation

\section{Introduction}

Simulated moving bed (SMB) technology developed by UOP in early 1960s [1] has emerged as a powerful tool for continuous countercurrent separation of binary mixtures of components with different adsorption affinities. Compared with batch chromatographic processes, the continuous SMB process reduces solvent consumption as much as 10 -fold and uses less column packing [2].

SMB separation technology has been extensively used for the large-scale fractionation of sugars and xylene isomers for more than 30 years. Recently, this technol-

\footnotetext{
* Corresponding author. Tel.: +351-22-508-1671; fax: +351-22508-1674.

E-mail address: arodrig@fe.up.pt (A.E. Rodrigues).
}

ogy has been brought into the field of fine chemistry and pharmaceutical industry [3].

The SMB process basic principle is a flow scheme that takes advantages of continuous and countercurrent movement of liquid and solid phase without actual movement of the adsorbent. This countercurrent movement is simulated by an appropriated flow switching sequence: the adsorbent bed is divided into a number of fixed bed columns, while the inlet and outlet lines move simultaneously one column at fixed time intervals in the direction of the liquid phase flow [4].

The modelling of chromatographic systems presents an interesting challenge since the process conditions are simple enough to allow a reasonably realistic mathematical description of the system. The first studies of the behaviour of SMB units through detailed models date back to the 1980s. The effect of changes in the operating parameters and in the layout of the unit has been 
analysed by modelling SMB units directly or by exploring the equivalence between SMB and true moving bed unit (TMB) [5]. Namely, two main strategies of modelling of SMB process can be distinguished in the open literature. The first one treats the SMB system as the equivalent true moving system. The second strategy represents the actual SMB configuration. Each of these two modelling strategies leads to models that can be different according to the description of the unit operation (staged or distributed plug flow system), the mass transfer resistance (equilibrium stage or mass transfer resistance within the fluid and/or solid phase) and adsorption equilibria (linear, Langmuir, bi-Langmuir or modified Langmuir). A review of these models can be found in Ruthven and Ching [6] and Pais [7].

The main difference between true moving bed and SMB units is their stationary regime: the TMB is designed to operate under steady state and the SMB under cyclic steady state, characterised by an identical transient concentration profiles during each period between two valve switches. The cyclic steady state (CSS) is particularly reached after a certain number of cycles, but the system state is still varying over the time because of the periodic movement of the inlet and outlet ports along the columns. Practically, increasing the number of the columns in the SMB system and decreasing their length, the SMB approaches the TMB system. Therefore, it is expected that the behaviour of SMB unit could be satisfactory predicted by considering it as an equivalent TMB unit just in the case of the SMB unit with sufficiently high number of columns [8-11].

The initial design and optimisation of the SMB processes is generally accomplished by reference to the equivalent TMB system [9-18]. The reasons for modelling of an equivalent unit instead of the real simulated moving unit lie into different level of difficulty involved in the solution of the two models and the required time for their computation. Since the true moving bed operates in steady state, the stationary behaviour is described by a set of ordinary differential and algebraic equations (DAEs). This is not the case for SMB, whereas the transient response requires the solution of a set of partial differential equations and algebraic equations. The cyclic steady state of SMB is normally calculated by running a dynamic simulation starting from given initial conditions and carrying out cycle by cycle until CSS is reached. Usually more than ten cycles are needed for the SMB to reach the cyclic steady state. The time required to compute stationary regime using simulated moving bed strategy is normally five to ten times longer than using equivalent true moving bed strategy [7].

In the last decade various methods were proposed in the aim of acceleration of CSS convergence. For instance, Ding and Le Van [19] proposed acceleration toward CSS for pressure swing adsorption (PSA) and temperature swing adsorption (TSA) processes using hybrid Newton-Broyden methods. Todd et al. [20] illustrated the advantage of node refinement as a useful tool for accelerating convergence to CSS of PSA and vacuum swing adsorption (VSA) processes. Smith and Westerberg [21] considered a shooting method that explicitly combines the CSS conditions within the process model. All these acceleration procedures are developed and applied in simulation of PSA/TSA/VSA processes, which could require thousands of cycles to achieve a CSS

Nilchan and Pantelides [22] developed a method for direct determination of CSS of rapid pressure swing adsorption (RPSA), in view of process optimisation. The method consists in replacing the initial conditions specification by a periodicity condition demanding that the system state at the end of each cycle is identical to that at its start. The system of partial differential equations and algebraic equations (PDAEs), which describes the RPSA behaviour, was reduced to a set of nonlinear algebraic equations (AEs) by simultaneous discretisation of both spatial and temporal variations. The orthogonal collocation on finite elements discretisation method is applied to the spatial domain and the second order backward finite difference method is applied to the temporal domain. The resulting system of algebraic equations is solved using a Newton type iterative method implemented in general Process Modelling System (gPROMS) [23].

Kloppenburg and Gilles [24] suggested a method for direct computation of periodic SMB state. A CSS is identified by the fact that the spatially distributed SMB unit state at the end of a switching time interval is identical to the state at the beginning of the interval, apart from a shift of exactly one column length. In order to directly compute the CSS the mathematical model is discretised with respect to time using trapezoidal rule. They used two alternative methods for the solution of model equations: (i) formulation of the model as a FORTRAN subprogram using DIVA code generator [25] and solution with the nonlinear equation solver NLEQ1S [26] and (ii) formulation of the model in AMPL [27] modelling language and solution with the solver LANCELOT [28]. The second alternative is more efficient in terms of computation time, since LANCELOT is specially designed for large-scale systems.

In this work two methods for direct cyclic steady state calculation, proposed by Nilchan and Pantelides [22] and Kloppenburg and Gilles [24], were applied in the prediction of cyclic steady state of SMB unit for 1,1'-bi2-naphthol enantiomers separation. The cyclic steady state was also predicted using the standard method for cyclic steady state determination, i.e. dynamic simulation starting from given initial conditions over a large number of cycles until the cyclic steady state is reached. Mathematical models were solved using the gPROMS [23] 
software package. Direct CSS predictions were compared with CSS predicted by dynamic simulation in terms of accuracy of SMB performance and computing time requirements.

\section{Mathematical modelling of SMB}

The SMB unit is constituted by a set of identical fixed bed columns connected in a series (Fig. 1). The inlet streams are the feed, which contains the binary mixture to be separated, and the eluent. The outlet streams are the extract, a mixture of more strongly adorbed component (A) and eluent, and the raffinate, which includes the less adsorbed component (B) and eluent. The counter current movement of the liquid and solid phases is simulated by shifting the inlet and outlet ports one column ahead in the direction of the fluid flow at regular time intervals (switching time period). Inlet and outlet points divide the unit in four sections, each of which performs a different function. Zone 1 is located between eluent and extract nodes and its purpose is to regenerate the adsorbent; zone 2 is between extract and feed nodes and its function is to desorb component $\mathrm{B}$, zone 3 is placed between feed and raffinate nodes and its function is to adsorb component A; zone 4 is located between raffinate and eluent nodes and its purpose is to regenerate the eluent.

The stationary regime of the SMB is a cyclic steady state, characterised by an identical transient concentration profiles during each period between two valve switches. The CSS is particularly reached after a certain number of cycles, but the system states are still varying over the time because of the periodic movement of the inlet and outlet ports along the columns. The correct determination of CSS is needed for the assessment of the SMB performance.

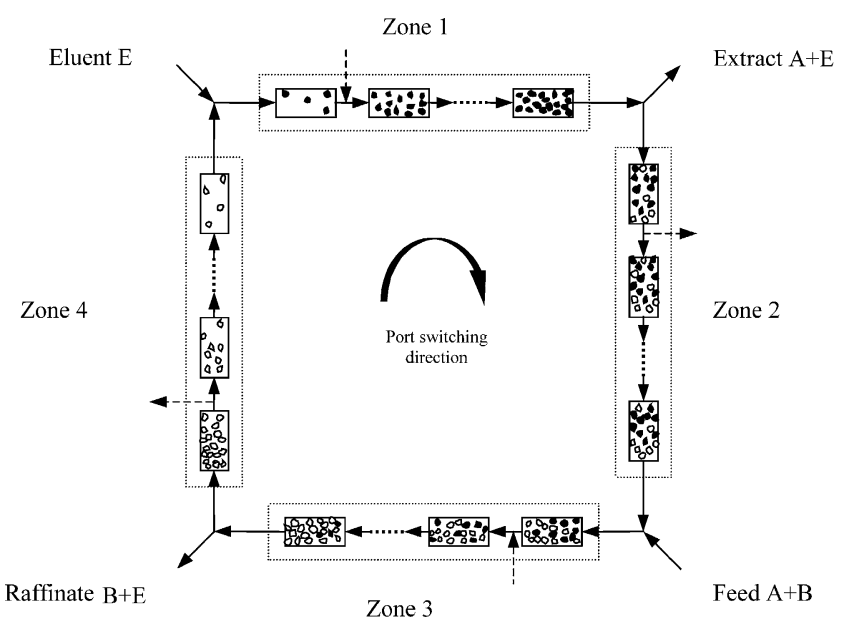

Fig. 1. Schematic diagram of SMB unit.
In this work the simulated moving bed strategy of modelling was used in the prediction of the stationary state of the SMB. In model formulation, the following assumptions have been considered.

- Negligible thermal effects;

- bed void fraction, radius and porosity of the particles are constant along the columns;

- constant flow rates in each section;

- negligible pressure drop;

- no radial variation occurs in the columns;

- axial dispersion flow for the liquid phase,

- the intraparticle mass transfer is described by a linear driving force (LDF) for the intraparticle mass transfer (i.e. parabolic intraparticle concentration profile)

The model equations are:

Mass balance in a volume element of the column $j$

$$
\begin{aligned}
& \varepsilon \frac{\partial c(i, j, z, t)}{\partial t}+(1-\varepsilon) \frac{\partial q(i, j, z, t)}{\partial t} \\
& \quad=\varepsilon D_{\mathrm{L}}(j) \frac{\partial^{2} c(i, j, z, t)}{\partial z^{2}}-\varepsilon v(j) \frac{\partial c(i, j, z, t)}{\partial z}
\end{aligned}
$$

where $i$ for component $(i=\mathrm{A}, \mathrm{B}), j$ for the column $(k=$ $1,2, \ldots . N)$

Particle mass balance

$\frac{\partial q(i, j, z, t)}{\partial t}=k(i)\left(q^{*}(i, j, z, t)-q(i, j, z, t)\right)$

Adsorption equilibrium isotherm

$q^{*}(i, j, z, t)=f(c(i, j, z, t))$

Boundary conditions

$z=0$

$c^{\text {in }}(i, j, t)=c(i, j, z, t)-\frac{D_{\mathrm{L}}(j)}{v(j)} \frac{\partial c(i, j, z, t)}{\partial z}$

$z=L \quad \frac{\mathrm{d} c(i, j, z, t)}{\mathrm{d} z}=0$

According to the scheme in Fig. 1, it appears that the SMB unit includes $N$ columns. Due to the switching of inlet and outlet ports, each column plays a different function during a whole cycle, depending on its location (zone). The complete model of the SMB unit is constituted by a set of mass balance equations for each of the $N$ columns, connected with each other by simple material balances at the connecting nodes. With each switching of the inlet and outlet ports, each column should be updated in terms of flowrate and inlet concentrations. The flowrate in each column, according to its location (zone), can be calculated by mass balance around the inlet and outlet nodes. The inlet concentration of each column is equal to the outlet concentration 
of the previous column $\left[c(i, j, t)^{\text {out }}=c(i, j+1, t)^{\text {in }}\right]$, except for the feed and eluent nodes.

Mass balances at the nodes of the inlet and outlet lines of the SMB

Eluent node:

$c(i, j, t)^{\text {out }} Q_{4}=c(i, j+1, t)^{\text {in }} Q_{1}$

Feed node:

$$
c(i, j, t)^{\text {out }} Q_{2}+c_{i, F} Q_{F}=c(i, j+1, t)^{\text {in }} Q_{3}
$$

From the mathematical point of view, the SMB processes can be classified as distributed parameter systems in which the dependent variables vary with respect to axial position as well as time. The SMB operation is described by a set of partial differential equations (mass balance equations) and algebraic equations (equilibrium isotherms and node mass balances). These PDAEs are highly coupled, which makes it impossible to obtain analytical solutions. Thus, the simulation of such processes relies on numerical methods employing space and time discretisation.

\section{Prediction of CSS of SMB}

In this work two approaches to cyclic steady state have been studied: the standard approach to CSS by dynamic simulation and the direct approach to CSS.

\subsection{Dynamic simulation of CSS}

The standard approach to CSS consist in carrying out dynamic simulation, starting from given initial conditions Eq. (8) and simulating cycle by cycle through successive substitution, where bed conditions from the previous cycle become initial conditions for the next cycle, continuing so forth until CSS is reached.

Initial conditions

$$
t=0 ; \quad c(i, j, z, t)=c_{0} \quad q(i, j, z, t)=q_{0}
$$

\subsection{Direct determination of CSS}

Two methods for direct CSS prediction have been used.

The first one, proposed by Nilchan and Pantelides [22], based on the fact that at cyclic steady state the conditions at the end of the cycle is identical to those at its beginning in both liquid and solid phases. Therefore, the initial conditions are replaced by the above periodicity conditions:

$$
\begin{gathered}
c(i, j, z, 0)=c\left(i, j, z, T_{\text {cycle }}\right) \quad i \in(A, B) \\
j \in(1,2, \ldots, N) \quad z \in(0, L)
\end{gathered}
$$

$$
\begin{gathered}
q(i, j, z, 0)=q\left(i, j, z, T_{\text {cycle }}\right) \quad i \in(A, B) \\
j \in(1,2, \ldots, N) \quad z \in(0, L)
\end{gathered}
$$

The second method for direct CSS prediction, proposed by Kloppenburg and Gilles [24], is based on the fact that at CSS the spatially distributed SMB unit state at the end of a switching time interval is identical to the state at the beginning of the interval, apart from a shift of exactly one column length. Therefore, the initial conditions are expressed as:

$$
\begin{aligned}
& c(i, j, z, 0)=c\left(i, j+1, z, t^{*}\right) \\
& \quad i \in(A, B) j \in(1,2, \ldots, N) z \in(0, L) \\
& q(i, j, z, 0)=q\left(i, j+1, z, t^{*}\right) \\
& \quad i \in(A, B) j \in(1,2, \ldots, N) z \in(0, L)
\end{aligned}
$$

Both methods are based on the same concept; the only difference is that the first method concerns the simulation of the whole cycle and the second one the simulation of just one switching time.

In the following sections the Nilchan and Pantelides [22] method for direct CSS prediction would be referred as Method 1 and Kloppenburg and Gilles method [24] as Method 2.

\subsection{Numerical considerations}

The dynamic model equations Eqs. (1)-(8) and direct CSS model equations (Eqs. (1)-(7) and (9) for Method 1 and Eqs. (1)-(7) and (10) for Method 2) were solved using the gPROMS [23], a software package for modelling and simulation of processes with both discrete and continuous as well as lumped and distributed characteristics. gPROMS is a high-level PDE package. No knowledge of programming language is necessary. The problems are described in gPROMs language, which allows symbolic specification of PDAEs, boundary conditions, initial conditions and appropriate coefficients. The system of PDAE is numericaly solved using the method of lines (MOL) [29]. This involves discretisation of the distributed equations with respect to all spatial domains, which results in a mixed set of time depended DAEs. gPRoms modelling language allows the user to specify the type of the spatial approximation method (e.g. finite difference or finite elements), as well as the granularity (e.g. number of the finite difference nodes, number of finite elements) and the order (e.g. first, second, etc.) of the approximation. The numerical discretisation is applied automatically. The resulting system of time depended DAEs can be solved by one of the integration codes implemented in gProms. More information on the package can be found in Barton and Pantelides [30] and Oh [31].

The dynamic model PDAE system Eqs. (1)-(8) was reduced into a set of ordinary DAEs in time by discretisation of the axial domain. A third order 
orthogonal collocation method in finite elements (OCFEM) comprising two internal collocation points was used for this purpose. The resulting system of DAE was integrated over time by employing DASOLV integration code. DASOLV is integrated in gPROMS, it is based on backward differentiation formulae, and automatically adjusts the time step size as well as the integration order to maintain the error of integration within a user specified tolerance. An absolute and relative tolerance of $10^{-5}$ was used.

In the case of the Method 1 and 2 for direct determination of CSS (Eqs. (1)-(7) and (9), and Eqs. (1) $-(7)$ and (10)) both space and temporal domains were discretised simultaneously. A third order OCFEM comprising two internal collocation points was used in the discretisation of spatial domain and a second order backward difference method (BFDM) was used for discretisation of temporal domain. The PDAE systems (Eqs. (1)-(7) and (9) and Eqs. (1)-(7) and (10)) are reduced directly into a set of nonlinear algebraic equations and solved using a Newton-type iterative method implement in gPROMS. The iterations were initialised with a chosen value for the concentration in the liquid $\left(0.8 \mathrm{~g} \mathrm{l}^{-1}\right)$ and solid phase $\left(2.45 \mathrm{~g} \mathrm{~g}^{-1}\right)$.

All simulations were performed on Pentium IV 1700 $\mathrm{MHz}$ processor with $1548 \mathrm{Mb}$ RAM memory.

\section{Simulation results and discussion}

The most recent SMB application is related with chiral technology. The separation of enantiomers is an important issue in various areas and particularly in the health-related field. It is well known that isomers can have different therapeutical effects and there is pressure of regulatory agencies for the separation of isomers [32].

In this work the chromatographic resolution of 1,1'bi-2-naphthol enantiomers was considered for the simulation purpose. The chiral stationary phase used in this system was 3,5-dinitrobenzoyl phenylglycine bonded to silica gel, and a mixture of $72 / 28(\mathrm{v} / \mathrm{v})$ heptane/isopropanol was used as eluent. The limit of solubility in this eluents is $3 \mathrm{~g}^{-1}$ of each enantiomer [7]. The adsorption equilibrium isotherms, measured at $25{ }^{\circ} \mathrm{C}$, are of biLangmuir type as proposed by the Separex group [33]:

$$
\begin{aligned}
q_{\mathrm{A}}^{*} & =\frac{3.73 c_{\mathrm{A}}}{1+0.0336 c_{\mathrm{A}}+0.0466 c_{\mathrm{B}}}+\frac{0.30 c_{\mathrm{A}}}{1+c_{\mathrm{A}}+3 c_{\mathrm{B}}} \\
q_{\mathrm{B}}^{*} & =\frac{2.69 c_{\mathrm{B}}}{1+0.0336 c_{\mathrm{A}}+0.0466 c_{\mathrm{B}}}+\frac{0.10 c_{\mathrm{B}}}{1+c_{\mathrm{A}}+3 c_{\mathrm{B}}}
\end{aligned}
$$

where $\mathrm{A}$ refers to the more-retained component, i.e. extract component and $\mathrm{B}$ to the less-retained component, i.e. raffinate component.

The operating conditions and model parameters used in the simulation study are presented in Table 1 .
Table 1

SMB operation conditions and model parameters

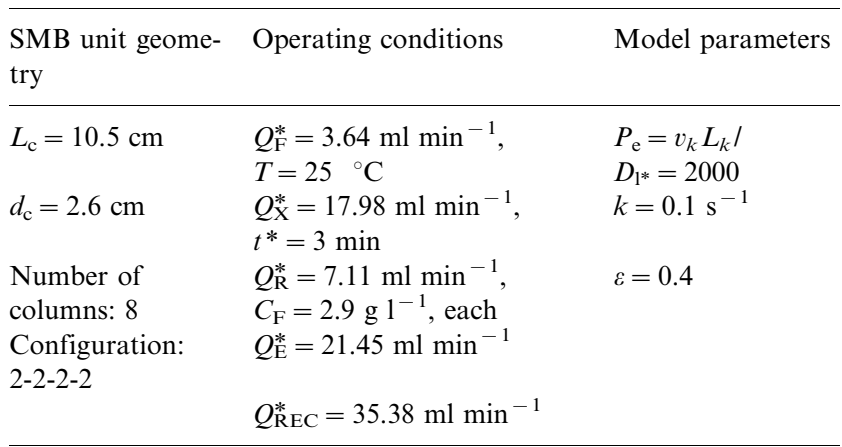

\subsection{Dynamic simulation of CSS}

First the cyclic steady state behaviour was simulated using the standard approach, i.e. cycle by cycle until reaching cyclic steady state. The model equations (Eqs. (1)-(8)) were solved starting from the initial conditions where no feed components are presented in the SMB unit, the columns are filled with eluent.

In order to determine when the steady state is achieved, the relative error was defined as sum of:

1) the relative error between the average concentrations (evaluated during a whole cycle) of each component in the extract (X) and raffinate (R) stream for the successive cycles:

$$
\begin{aligned}
(\mathrm{X}+\mathrm{R}) & \text { relative error } \% \\
= & 100\left(\frac{\left|\bar{c}_{\mathrm{X}_{k}}^{\mathrm{A}}-\bar{c}_{\mathrm{X}_{k-1}}^{\mathrm{A}}\right|}{\bar{c}_{\mathrm{X}_{k}}^{\mathrm{A}}}+\frac{\left|\bar{c}_{\mathrm{X}_{k}}^{\mathrm{B}}-\bar{c}_{\mathrm{X}_{k-1}}^{\mathrm{B}}\right|}{\bar{c}_{\mathrm{X}_{k}}^{\mathrm{B}}}\right) \\
& +\left(\frac{\left|\bar{c}_{\mathrm{R}_{k}}^{\mathrm{A}}-\bar{c}_{\mathrm{R}_{k-1}}^{\mathrm{A}}\right|}{\bar{c}_{\mathrm{R}_{k}}^{\mathrm{A}}}+\frac{\left|\bar{c}_{\mathrm{R}_{k}}^{\mathrm{B}}-\bar{c}_{\mathrm{R}_{k-1}}^{\mathrm{B}}\right|}{\bar{c}_{\mathrm{R}_{k}}^{\mathrm{B}}}\right)
\end{aligned}
$$

The comparison of the extract and raffinate average concentrations in two consecutive cycles, instead of comparison of their concentration histories was considered, since there was no significant difference between the relative errors calculated in both ways and

2) the mass balance (MB) relative error, i.e. relative error between the total amount of each component that enters (in the feed stream) and leaves (in the extract and raffinate stream) the system (evaluated during a full cycle):

MB relative error $\%$

$$
\begin{gathered}
=100 \frac{\left|Q_{\mathrm{F}} c_{\mathrm{F}}^{\mathrm{A}}-\left(Q_{\mathrm{X}} \bar{c}_{\mathrm{X}}^{\mathrm{A}}+Q_{\mathrm{R}} \bar{c}_{\mathrm{R}}^{\mathrm{A}}\right)\right|}{Q_{\mathrm{F}} c_{\mathrm{F}}^{\mathrm{A}}} \\
+\frac{\left|Q_{\mathrm{F}} c_{\mathrm{F}}^{\mathrm{B}}-\left(Q_{\mathrm{X}} \bar{c}_{\mathrm{X}}^{\mathrm{B}}+Q_{\mathrm{R}} \bar{c}_{\mathrm{R}}^{\mathrm{B}}\right)\right|}{Q_{F} c_{\mathrm{F}}^{\mathrm{B}}}
\end{gathered}
$$

A relative error of $1 \%$ was considered since this 
could provide the estimation of the SMB performances with satisfactory accuracy and acceptable computing time.

First of all the minimum number of the axial domain elements, i.e. number of finite elements per column used in OCFEM, which enables CSS prediction satisfying the relative error defined above was defined. Some simulations were carried out, starting with three elements per column, and increasing the number of finite elements until eight. CSS was achieved in 13 cycles. After 20 cycles the relative error approach to zero. Table 2 shows the average extract and raffinate concentrations calculated over the whole cycle at CSS, the corresponding extract and raffinate purities, the relative error and CPU times needed for dynamic simulation of CSS using different number of finite elements per column. It can be seen that (i) for the all number of elements used the relative error was less than $1 \%$ and (ii) there was no significant advantage of using more finite elements per column, in terms of extract and raffinate concentrations and their purities. Therefore, three subdivisions per column for the axial domain were selected for the further simulation studies.

\subsection{Direct determination of CSS}

The Methods 1 and 2 for direct determination of CSS require simultaneous discretisation of axial and temporal domain. A third orthogonal collocation method was applied for discretisation of spatial domain using the selected number of finite elements (3) from the dynamic simulation study. A second order BFDM was used for the discretisation of the temporal domain.

First, the CSS was predicted by Method 1, simulating one cycle and requiring that the conditions at the end of the cycle are identical to those at its beginning in both liquid and solid phases (Eq. (9)). Some simulations were carried out in order to determine the number of finite elements in the BFDM that enables prediction of CSS within the considered relative error $(1 \%)$. The simulations were initialised with time step $0.3 \mathrm{~min}$ (cycle time divided in 80 finite elements) until time step $0.1667 \mathrm{~min}$ (cycle time divided in 144 finite elements). The maximum number of time steps tested was practically the maximum number of time steps supported by the computer performance.

Since the methods for direct determination of CSS predict the stationary state directly, the relative error is calculated only by the Eq. (14). The relative error and CPU time for different time steps are presented in Table 3 and Fig. 2. It can be seen that computing time increases significantly with increasing the number of the finite elements for the time domain. The relative error shows linear tendency of decreasing with increasing the number of the finite elements and is expected to further decrease with increasing the number of finite elements per column.

The above procedure was also followed in the direct CSS prediction using Method 2. In this case the process was simulated during just one switching time requiring that the spatially distributed SMB unit state at the end of a switching time interval is identical to the state at the beginning of the interval, apart from a shift of exactly one column length (Eq. (10)). The simulations were initialised with time step $0.3 \mathrm{~min}$ (switching time divided in ten finite elements) until time step 0.125 min (switching time divided in 24 finite elements). The relative error and CPU time for different time steps are presented in Table 4. The CSS within relative error of $1 \%$ could be determined using at least ten finite elements $(0.3 \mathrm{~min}$ time step) for the time domain.

\subsection{Comparison of dynamic simulation and direct determination of CSS}

The dynamic CSS prediction and both methods for direct CSS prediction were compared in terms of: concentration profiles, extract and raffinate histories, extract and rafinate purities and CPU times. For the comparison following simulation runs were selected:

i) dynamic simulation of CSS (three elements per column).

Table 2

Relative error and CPU time for different number of finite elements for the spatial domain-prediction of CSS by dynamic simulation

\begin{tabular}{|c|c|c|c|c|c|c|c|c|}
\hline \multirow[t]{2}{*}{ Number of finite elements } & \multicolumn{2}{|c|}{$\begin{array}{l}\text { Average } X \text { concentration } \\
\left(\mathrm{g} \mathrm{l}^{-1}\right)\end{array}$} & \multicolumn{2}{|c|}{$\begin{array}{l}\text { Average } \mathrm{R} \text { concentration } \\
\left(\mathrm{g} \mathrm{l}^{-1}\right)\end{array}$} & \multirow[t]{2}{*}{ X purity $(\%)$} & \multirow[t]{2}{*}{ R purity (\%) } & \multirow[t]{2}{*}{ Relative error $(\%)$} & \multirow[t]{2}{*}{ CPU time (s) } \\
\hline & A & $\mathrm{B}$ & A & $\mathrm{B}$ & & & & \\
\hline 3 & 0.5798 & 0.0257 & 0.0183 & 1.4124 & 95.75 & 98.72 & 0.83 & 32 \\
\hline 4 & 0.5796 & 0.0256 & 0.0188 & 1.4125 & 95.77 & 98.69 & 0.85 & 39 \\
\hline 5 & 0.5796 & 0.0256 & 0.0190 & 1.4126 & 95.77 & 98.67 & 0.86 & 48 \\
\hline 6 & 0.5795 & 0.0255 & 0.0192 & 1.4126 & 95.78 & 98.66 & 0.86 & 57 \\
\hline 7 & 0.5795 & 0.0255 & 0.0192 & 1.4126 & 95.78 & 98.66 & 0.87 & 67 \\
\hline 8 & 0.5795 & 0.0255 & 0.0193 & 1.4126 & 95.78 & 98.65 & 0.87 & 80 \\
\hline
\end{tabular}


Table 3

Relative error and CPU time for different number of finite elements for the temporal domain in the direct CSS prediction using Method 1

\begin{tabular}{|c|c|c|c|}
\hline Number of finite elements & Time step (min) & Relative error $(\%)$ & CPU time (s) \\
\hline 80 & 0.3000 & 8.85 & 2279 \\
\hline 96 & 0.2500 & 7.22 & 3807 \\
\hline 104 & 0.2308 & 6.70 & 5669 \\
\hline 120 & 0.2000 & 5.83 & 9912 \\
\hline 128 & 0.1875 & 5.50 & 10451 \\
\hline 144 & 0.1666 & 4.91 & 15812 \\
\hline
\end{tabular}

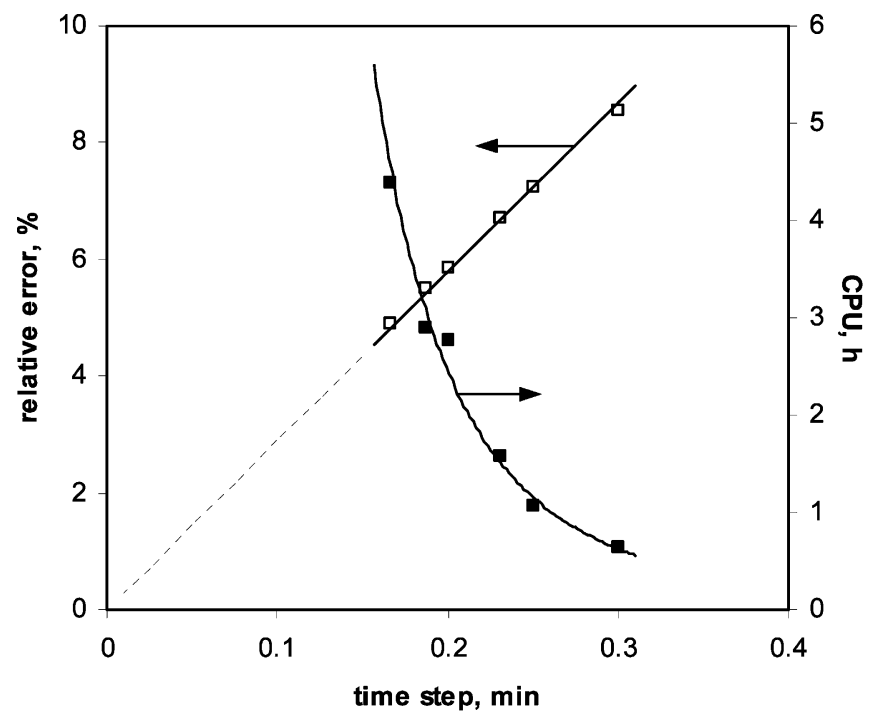

Fig. 2. Relative error and CPU time vs. time step used in temporal discretisation in the case of direct prediction of CSS by Method 1 ($\diamond-$ relative error, - - - CPU time).

ii) Method 1 for direct CSS prediction (three elements per column, 144 elements for the cycle time-corresponding to 0.1667 min time step).

iii) Method 2 for direct CSS prediction (three elements per column, 18 elements for the switching time, corresponding to $0.1667 \mathrm{~min}$ time step).

The CSS internal concentration profiles calculated by (i) dynamic simulation; (ii) Method 1 and (iii) Method 2 are presented in Fig. 3. The corresponding cyclic steady state extract and raffinate concentration histories during one switching time are presented in Fig. 4.
There is no significant difference in the prediction of CSS concentration profiles using the standard dynamic approach and both direct CSS approaches. The more significant difference could be observed in the extract and raffinate histories predicted directly using Method 1 (Fig. 4a and b) and those predicted by dynamic simulation and Method 2. This was expected since the relative error in the case of direct CSS prediction using Method 1 is significant (around 5\%).

The extract and raffinate purities calculated from the selected simulation runs are presented in Table 5. The relative error between the extract and raffinate purities determined by the dynamic simulation and the methods for direct CSS prediction was calculated using the following equation:

relative error $\%=100$

$\underline{\mid \text { (dynamic simulation })-(\text { direct CSS simulation }) \mid}$

The values of the extract and raffinate relative errors are presented in Table 5. It could be seen that there is small relative difference between the extract and raffinate purities calculated by both approaches (dynamic approach and direct CSS approach). Since in both approaches the axial domain is discretised using the same method, third order OCFEM, and the same number of finite elements (3), the relative error between the dynamic simulation and the methods for direct cyclic steady state prediction arise mainly from the discretisation of the temporal domain.

Table 4

Relative error and CPU time for different number of finite elements for the temporal domain in the direct CSS prediction using Method 2

\begin{tabular}{llll}
\hline Number of finite elements & Time step $(\mathrm{min})$ & Relative error (\%) & CPU time (s) \\
\hline 10 & 0.3000 & 0.84 & 6 \\
12 & 0.2500 & 0.79 & 12 \\
13 & 0.2308 & 0.76 & 14 \\
16 & 0.1875 & 0.68 & 19 \\
18 & 0.1667 & 0.63 & 48 \\
20 & 0.1500 & 0.59 & 68 \\
24 & 0.1250 & 0.52 & 86 \\
\hline
\end{tabular}




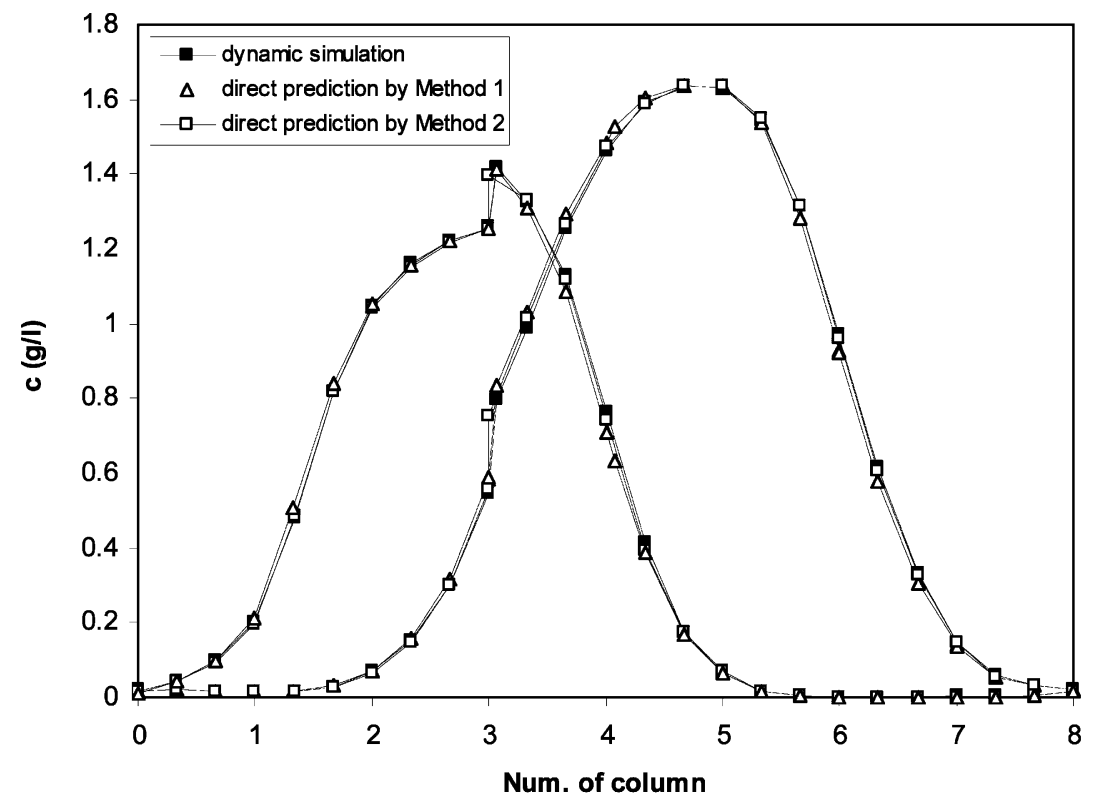

Fig. 3. Cyclic steady state concentration profiles obtained by: $-\mathbf{-}-$ dynamic simulation, $-\Delta-$ Method 1 and $-\square-$ Method 2 .

The comparison of the CPU times (Table 5) shows that dynamic simulation and Method 2 require similar computation time for prediction of CSS. We need to take into consideration that the simulation run for Method 2 chosen for the comparison predicts the CSS with relative error $0.63 \%$ (Table 4) and dynamic simulation with relative error $0.83 \%$. If we compare the dynamic simulation CSS prediction with CSS predicted with Method 2 for similar relative error (around $0.8 \%$ ), we can see that Method 2 (time step $0.3 \mathrm{~min}$, Table 5, values in brackets) requires around 5 times less computation time than dynamic simulation (32 s CPU for dynamic simulation of CSS, against $6 \mathrm{~s}$ CPU time for Method 2 for direct prediction of CSS). The Method 2 for direct prediction of CSS would be even more competitive in the cases of dynamic simulation where more cycles are needed to achieve the cyclic steady state of a SMB unit.

There is high difference in the CPU times required for direct prediction of CSS using Method 1 and those from dynamic simulation and direct prediction using Method 2. Part of the explanation arises from the special treatment of the time domain in gPROMS. All variables are assumed to be distributed with respect to time without being declared explicitly in the model. The numerical treatment of the time domain in dynamic simulation is different from that of the other distribution domains, but there is also the possibility in gPROMS to treat the time as just another distribution domain declared and handled explicitly [31]. This option was used for the direct determination of CSS. Both the computer memory and the computation cost required by the explicit handling of the time domain are likely to be higher than those associated with the implicit alterna- tive. Also the guaranteed control of the temporal discretisation error is lost [31]. There is still enormous difference in the computation time required for direct prediction of CSS using Method 1 and Method 2. In both methods the same time step was used $(0.1667 \mathrm{~min})$ that implies ratio 8:1= number of ODE's in Method 1/ number of ODE's in Method 2, since in Method 1 one whole cycle is simulated and in Method 2 only one switching time. It was shown before that the computing time increases significantly with increasing the number of the finite elements for the time domain (Fig. 2). Therefore, linearity between number of ODE's and computation time for Method 1 and 2 should not be expected.

However, Method 1 may be advantageous in certain circumstances, as the PSA/VSA/TSA processes where one or two adsorption columns are normally used and thousands of cycles could be needed for achieving CSS [22].

Dynamic simulation could be more competitive in the study of the effect of some model parameters. Namely, if all planned simulations runs (comprising different values of examined model parameter) are carried out as a sequence, using as initial conditions the CSS conditions of the previous simulation. To illustrate this a sequence of dynamic simulation using different switching times was carried out. The operation conditions used in the simulation sequence are those from Table 1, except the switching time. The switching time was increased starting from $t^{*}=2.7 \mathrm{~min}$ until $t^{*}=3.1 \mathrm{~min}$, with step of $0.1 \mathrm{~min}$. In the first dynamic CSS simulation $\left(t^{*}=2.7 \mathrm{~min}\right)$ the initial conditions where no feed components are presented in the SMB unit were used. In the next simulation $\left(t^{*}=2.8 \mathrm{~min}\right)$ the CSS concen- 


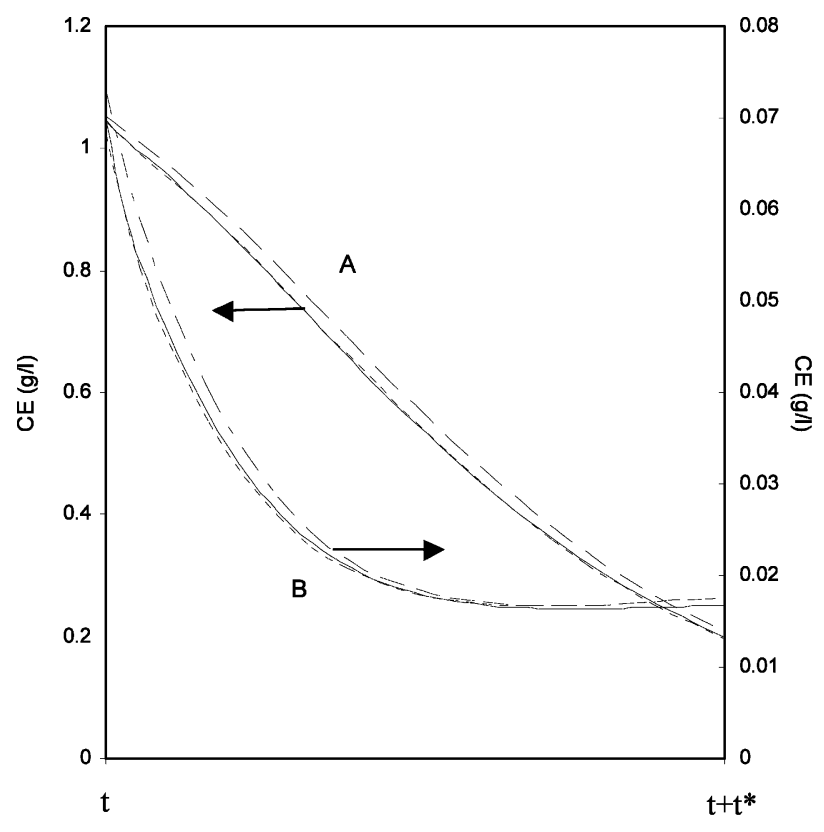

a)

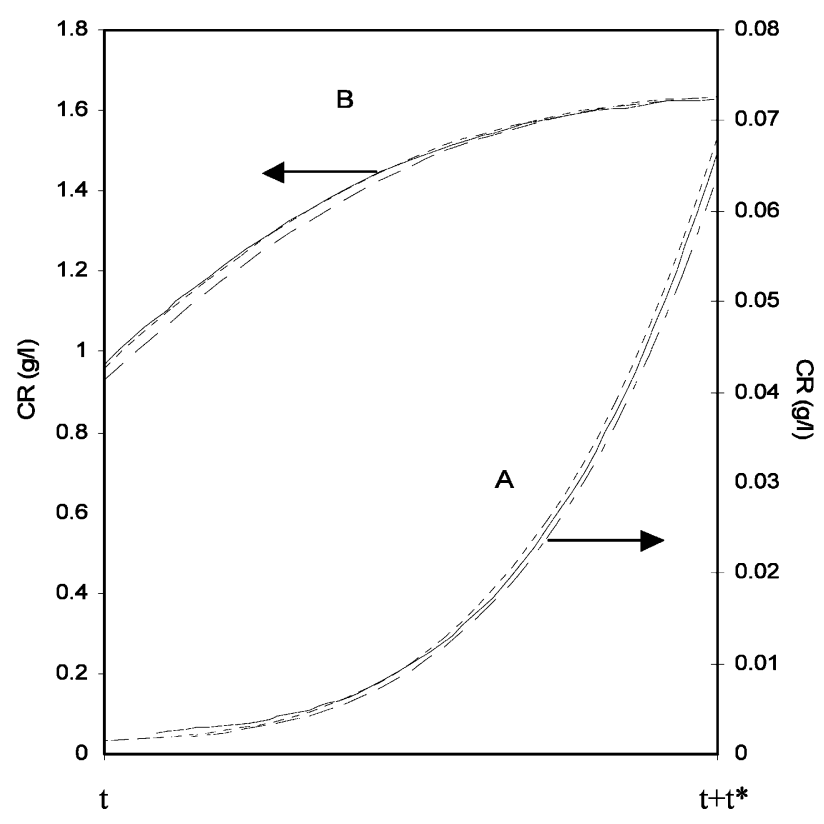

b)

Fig. 4. Concentration histories in (a) the extract and (b) raffinate at cyclic steady state, during one switching time period, _ — — dynamic

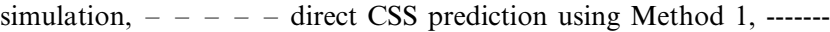
direct CSS prediction using Method 2.

tration profiles in liquid and solid of previous simulation were imposed as initial conditions. All sequence was carried out in this manner. The dynamic simulations of CSS for all switching times were repeated for the initial condition of no feed component present in the SMB unit. The comparison results are presented in Table 6. It could be seen that using the CSS conditions as initial conditions in the sequential study leads to a decrease in cycles needed to reach CSS by 4 . In this kind of studies the knowledge of the influence of the model parameters is essential in the choice of: the interval in which the particular model parameter would be varied, the amplitude of the change and the direction of the change, i.e. increasing or decreasing the value of the model parameter in the chosen interval of variation. Just for illustration, if we use the CSS corresponding to $t^{*}=$ $3.5 \mathrm{~min}$ as initial condition in the prediction of CSS corresponding to $t^{*}=3 \mathrm{~min}$, more cycles would be needed to reach the CSS than when we start the simulation with null initial conditions (big step change of $t^{*}$ and wrong direction of change of $t^{*}$ ). In the case of $t^{*}=3.5$ the SMB operates out of the separation region, i.e. no pure extract and raffinate, which results from the shift of the extract and raffinate component concentration profiles in undesirable direction.

This procedure cannot be generalised for all process parameters. For instance, the procedure is not efficient in the study of the effect of mass transfer parameter $(k)$ on the SMB process performance. Increasing the mass transfer parameter the concentration profiles become steeper and fine grids should be used in the discretisation of the axial domain for accurate prediction of CSS. On the other hand one of the requirements of the sequential simulations is the use of the same number of finite elements in all simulations.

For example, in the case of mass transfer parameter $k=6 \min ^{-1}$ three elements per column are needed; in the case of $k=12 \mathrm{~min}^{-1}$, ten elements per column and in the case of $k=18 \mathrm{~min}^{-1}, 15$ per column are needed to simulate CSS within a relative error of $1 \%$. In Table 7 the CPU time and the number of the cycles needed to achieve CSS for mass transfer parameter $k=12 \mathrm{~min}^{-1}$ (i) using null initial conditions for ten elements (coarse grid) and 15 elements (fine grid) per column; and (ii) using CSS of the simulation run corresponding to $k=6$ $\min ^{-1}$ (coarse grid) and CSS corresponding to $k=18$ $\min ^{-1}$ (fine grid) are presented. It could be seen that less CPU time consuming is the case where the CSS is predicted using the CSS profiles of the simulation run for $k=6 \mathrm{~min}^{-1}$. The initialisation of the CSS dynamic simulation using CSS corresponding to $k=18 \mathrm{~min}^{-1}$ would decreases the number of cycles needed to archive the CSS, but also require longer computation time, since more fine elements have to be used.

\section{Conclusions}

Cyclic steady state of SMB 1,1'-bi-2-naphthol enatiomers separation has been computed using two modelling approaches: (i) the standard approach, i.e. dynamic simulation cycle by cycle till steady state is achieved; and (ii) direct approach to CSS. 
Table 5

Cyclic steady state SMB performance, CPU time and relative error for various prediction methods

\begin{tabular}{|c|c|c|c|c|c|}
\hline & \multicolumn{2}{|l|}{ Extract } & \multicolumn{2}{|l|}{ Raffinate } & \multirow[t]{2}{*}{$\mathrm{CPU}(\mathrm{s})$} \\
\hline & Purity $(\%)$ & $\begin{array}{l}\text { Relative error }(\%) \\
\text { (Eq. (15)) }\end{array}$ & Purity $(\%)$ & $\begin{array}{l}\text { Relative error }(\%) \\
\text { (Eq. (15)) }\end{array}$ & \\
\hline Dynamic simulation & 95.75 & - & 98.72 & - & 32 \\
\hline Direct CSS prediction by Method 1 time step $=0.1667 \mathrm{~min}$ & 95.62 & 0.14 & 98.75 & 0.03 & 15812 \\
\hline $\begin{array}{l}\text { Direct CSS prediction by Method } 2 \text { time step }=0.1667 \mathrm{~min} \\
\text { (time step }=0.3 \mathrm{~min} \text { ) }\end{array}$ & $95.71(95.66)$ & $0.04(0.09)$ & $98.67(98.61)$ & $0.05(0.11)$ & $48(6)$ \\
\hline
\end{tabular}

Two methods were used for direct prediction of CSS. Method 1 is based on the substitution of the initial condition by the periodicity condition, i.e. the system state at the end of each cycle is identical to that at its start. Method 2 is based on the fact that at CSS the spatially distributed SMB unit state at the end of a switching time interval is identical to the state at the beginning of the interval, apart from a shift of exactly one column length. Both methods are based on the same concept; the only difference is that the first method concerns simulation of whole cycle and the second deals with simulation of just one switching time period.

gPROMS was used for modelling and simulation of dynamic simulation and direct CSS prediction approaches. In the case of the methods for direct CSS prediction spatial and temporal domain were discretised simultaneously.

Both approaches were compared in terms of accuracy in the prediction of SMB unit performance and computation time.

Method 2 for direct CSS prediction requires less computation time than the dynamic simulation of CSS. The cyclic steady state SMB performances were calculated in few seconds with high accuracy. This method could be used as efficient tool for fast prediction of CSS especially in the cases of SMB units that require more cycles to achieve CSS.

Method 1 for direct prediction of CSS, based on substitution of initial conditions with periodicity conditions, is more time consuming than the dynamic simulation approach for this application. It should be stressed that in the case of cyclic processes with short cycle time, such as PSA/VSA where thousands of cycles are needed to reach CSS, this method is of importance.

Dynamic simulation of CSS of SMB processes is sometimes more competitive if several related simulations are carried out as a sequence. This could be used in the study of the effect of model parameters, if the series of simulations comprising different values of the examined model parameter is carried out as sequence using as initial conditions in each simulation the CSS of the previous simulation.

The development of new efficient numerical algorithms for the solution of large systems of nonlinear algebraic equations will certainly contribute to make the direct CSS even more competitive to the standard method for CSS prediction of SMB processes.

\section{Acknowledgements}

We gratefully acknowledge the useful and fruitful suggestions of one of the anonymous reviewers. Mirjana Minceva acknowledges the financial support of 'Fundação para a Ciência e Tecnologia', grant PRAXIS XXI/ BD/19503/99.

\section{Appendix A: Nomenclature}

C fluid phase concentration $\left(\mathrm{kg} \mathrm{m}^{-3}\right)$

$c^{\text {out }} \quad$ fluid phase concentration at the outlet of column $\left(\mathrm{kg} \mathrm{m}^{-3}\right)$

Table 6

Sequential study of the effect of switching time on the number of cycles to reach CSS by dynamic simulation ${ }^{\mathrm{a}}$

\begin{tabular}{|c|c|c|c|c|}
\hline \multirow[t]{2}{*}{$t^{*}(\min )$} & \multicolumn{2}{|c|}{ Using the $c_{i j}=q_{i j}=0$ initial condition } & \multicolumn{2}{|c|}{ Using the CSS profiles of previous simulation } \\
\hline & Number of cycles to reach CSS & Relative error $(\%)$ & Number of cycles to reach CSS & Relative error $(\%)$ \\
\hline 2.7 & 13 & 1.00 & - & - \\
\hline 2.8 & 12 & 0.87 & 8 & 0.83 \\
\hline 2.9 & 11 & 0.81 & 7 & 0.91 \\
\hline 3.0 & 13 & 0.83 & 9 & 0.79 \\
\hline 3.1 & 17 & 0.93 & 13 & 0.99 \\
\hline
\end{tabular}

\footnotetext{
a Three finite elements per column.
} 
Table 7

Influence of the type of the initial conditions on the number of the cycles needed to reach CSS and the computation time by dynamic simulation for $k=12 \mathrm{~min}^{-1}$

\begin{tabular}{|c|c|c|c|c|}
\hline \multirow[t]{3}{*}{ Type of the grid used in discretisation of axial domain } & \multicolumn{4}{|c|}{ Type of the initial conditions } \\
\hline & \multicolumn{2}{|c|}{ Null initial conditions } & \multicolumn{2}{|c|}{ CSS profiles of previous simulation } \\
\hline & cycles & CPU (s) & Cycles & CPU (s) \\
\hline Coarse grid (10 elements per column) & 15 & 104 & $12^{\mathrm{a}}$ & 92 \\
\hline Fine grid ( 15 elements per column) & 15 & 174 & $11^{\mathrm{b}}$ & 142 \\
\hline
\end{tabular}

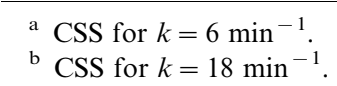

$c^{\text {in }} \quad$ fluid phase concentration at the inlet of

column $\left(\mathrm{kg} \mathrm{m}^{-3}\right)$

$D_{\mathrm{L}} \quad$ axial dispersion coefficient $\left(\mathrm{m}^{2} \mathrm{~s}^{-1}\right)$

$d_{\mathrm{c}} \quad$ column diameter (m)

$k \quad$ intraparticle mass-transfer coefficient $\left(\min ^{-1}\right)$

$L_{\mathrm{c}} \quad$ column length $(\mathrm{m})$

$Q \quad$ fluid flow-rate $\left(\mathrm{m}^{3} \mathrm{~s}^{-1}\right)$

$q \quad$ average adsorbed phase concentration $(\mathrm{kg}$ $\mathrm{m}^{-3}$ )

$q^{*} \quad$ adsorbed phase concentration in equilibrium with $c\left(\mathrm{~kg} \mathrm{~m}^{-3}\right)$

$t \quad$ time (s)

$t^{*} \quad$ switching time (s)

$T_{\text {cycle }} \quad$ cycle time (s)

$v \quad$ interstitial fluid velocity $\left(\mathrm{m} \mathrm{s}^{-1}\right)$

$Z \quad$ axial coordinate (m)

$\varepsilon \quad$ bed porosity, dimensionless

Subscripts and superscripts

$i \quad$ adsorbable components $(i=\mathrm{A}, \mathrm{B})$

$j \quad$ number of column $(j=1,2, \ldots \ldots N)$

E, X, F, eluent, extract, feed and raffinate streams $\mathrm{R}$

\section{References}

[1] D.B. Broughton, C.G. Gerhold, US Pat. 2,985,589 (1961).

[2] R.-M. Nicoud, R.E. Majors, Simulated moving bed chromatography for preparative separations, LCGC 18 (2000) 680.

[3] J.E. Rekoske, Chiral separation, AIChE J. 47 (2001) 2.

[4] L.S Pais, J.M. Loureiro, A.E. Rodrigues, Separation of enentiomers of a chiral epoxide by simulated moving bed chromatography, J. Chromatogr. A 827 (1998) 215.

[5] C. Migliorini, A. Gentilini, M. Mazzotti, M. Morbidelli, Design of simulated moving bed units under nonideal conditions, Ind Eng. Chem. Res. 38 (1999) 2400.

[6] D.M. Ruthven, C.B. Ching, in: G. Ganetsos, P.E. Barker (Eds.), Preparative and Production Scale Chromatography, Marcel Dekker, New York, 1993, p. 629.

[7] L.S. Pais, Chiral separation by simulated moving bed chromatography, Ph.D.Thesis, Faculy of Engineering, University of Porto, 1998.
[8] K. Hidajat, C.H. Ching, D.M. Ruthven, Simulated countercurrent adsorption processes: a theoretical analysis of the effect of subdividing the adsorbed bed, Chem. Eng. Sci. 41 (1986) 2953.

[9] D.M. Ruthven, C.H. Ching, Counter-current and simulated counter-current adsorption separation processes, Chem. Eng. Sci. 44 (1989) 1011.

[10] K.H. Chu, M.A. Hashim, Simulated counter-current adsorption processes: a comparison of modelling strategies, Chem. Eng. J. 56 (1995) 59.

[11] L.S. Pais, J.M. Loureiro, A.E. Rodrigues, Modelling strategies for enantiomers separation by SMB chromatography, AIChE J. 44 (1998) 561.

[12] D.C.S Azevedo, A.E. Rodrigues, Design of a simulated moving bed in the presence of mass-transfer resistance, AIChE J. 45 (1999) 956.

[13] Z. Ma, N.-H.L., Standing wave analysis of SMB chromatography: linear systems, AIChE J. 43 (1997) 2488

[14] A.S.T. Chiang, Continuous chromatographic process based on SMB technology, AIChE J. 44 (1998) 1930.

[15] G. Storti, M. Masi, S. Carra, M. Morbidelli, Optimal design of multicomponent counter-current adsorption separation processes involving nonlinear equilibria, Chem. Eng. Sci. 44 (1989) 1329.

[16] G. Storti, R. Baciocchi, M. Mazzoti, M. Morbidelli, Design of optimal operating conditions of simulated moving bed adsorptive separation units, Ind. Eng. Chem. Res. 34 (1995) 288.

[17] M. Mazzotti, G. Storti, M. Morbidelli, Robust design of countercurrent adsorption separation processes. 2. Multicomponent systems, AIChE J. 40 (1994) 1825.

[18] M. Mazzotti, G. Storti, M. Morbidelli, Optimal operation of simulated moving bed units for nonlinear chromatographic separations, J. Chromatogr. A 769 (1997) 3.

[19] Y. Ding, M.D. Le Van, PSA and TSA simulations: enhancements for accelerated convergence to the periodic state, AIChE Annual Meeting, Los Angeles, CA, 13-17 November 2000; Paper 158g.

[20] R.S. Todd, J. He, P.A. Webley, C. Beh, S. Wilson, M.A. Lloyd, Fast finite-volume method for PSA/VSA cycle simulation-experimental validation, Ind. Eng. Chem. Res. 40 (2001) 3217.

[21] O.J. Smith, A.W. Westerberg, Acceleration of cyclic steady-state convergence for ressure swing adsorption models, Ind. Eng. Chem. Res. 31 (1992) 1569.

[22] S. Nilchan, C. Pantelides, On optimization of periodic adsorption processes, Adsorption 4 (1998) 113.

[23] gProms v1.7 User Guide, Process System Enterprise Ltd., London, 1999.

[24] E. Kloppenburg, E.D. Gilles, A new concept for operating simulated moving-bed processes, Chem. Eng. Tech. 22 (1999) 813.

[25] K.D. Mohl, A. Spieker, E. Stein, E. Gilles, in: A. Kuhn, S. Wenzel (Eds.), DIVA-Eine Umgenbung zur Simulation, Analyse und Optimierung verfahrenstechnischer Prozesse, Simulationstechnik. ASIM-Symposium in Dortmund, vol. 11, Veiweg Verlag, Braunschweig/Wiesbaden, 1997, pp. 278-283. 
[26] P. Deuflhard, U. Nowak, M. Wulkow, Recent developments in chemical computing, Comp. Chem. Eng. 14 (1990) 1249.

[27] R. Fourer, D.M. Kernighan, B.W. Kernighan, AMPL. A Modeling Language for Mathematical Programming, Boyd \& Fraser Publishing Company, 1993.

[28] A.R. Conn, LANCelot-A fortran Package for Large-Scale Nonlinear Optimization (Release A), 1992.

[29] W.E. Schiesser, The Numerical Method of Lines, Academic Press, New York, 1991.

[30] P.I. Barton, Pantelides, Modeling of combined discrete/continuous processes, AIChE J. 40 (1994) 966.
[31] M. Oh, Modelling and simulation of combined lumped and distributed processes, Ph.D. Thesis, University of London, 1995.

[32] R.A. Sheldon, Chirotechnology: Industrial Synthesis of Optically Active Compounds, Marcel Dekker, New York, USA, 1993.

[33] R.M. Nicoud, A. Seidel-Morgenstern, Adsorption isotherms: experimental determination and application to preparative chromatography, in: R.M. Nicoud (Ed.), Simulated Moving Bed Basics and Applications, Institut Nacional Politechnique de Lorraine, Nancy, France, 1993. 